\title{
Treatment of locally advanced pancreatic cancer by percutaneous and intraoperative irreversible electroporation: general hospital cancer center experience
}

\author{
L. LAMBERT ${ }^{1, *}$, J. HOREJS ${ }^{1}$, Z. KRSKA ${ }^{2}$, D. HOSKOVEC ${ }^{2}$, L. PETRUZELKA ${ }^{3}$, T. KRECHLER ${ }^{4}$, P KRIZ ${ }^{5}$, J. BRIZA
}

${ }^{1}$ Department of Radiology, First Faculty of Medicine, Charles University in Prague; ${ }^{2}$ First Department of Surgery, First Faculty of Medicine, Charles University in Prague; ${ }^{3}$ Department of Oncology, First Faculty of Medicine, Charles University in Prague; ${ }^{4}$ Fourth Department of Medicine, First Faculty of Medicine, Charles University in Prague; ${ }^{5}$ Department of Anaesthesiology, Resuscitation and Intensive Medicine, First Faculty of Medicine, Charles University in Prague

*Corresponding author: lambert.lukas@gmail.com

Received June 11, 2015 / Accepted September 14, 2015

\begin{abstract}
The aim of this study was to evaluate the safety of irreversible electroporation (IRE) and the outcome of patients undergoing IRE of locally advanced pancreatic cancer (PC). Twenty-one patients with unresectable PC underwent open ( $\mathrm{n}=19)$ or percutaneous $(n=2)$ IRE of the tumor using the Nanoknife system with two electrodes that were repositioned several times to affect the whole mass. The size of the tumor was $39 \pm 10 \mathrm{~mm}$ with a range from 21 to $65 \mathrm{~mm}$. Five patients underwent neoadjuvant chemotherapy and seven patients were treated with chemotherapy after IRE. Complications occurred in five patients, which resulted in prolongation of the average hospital stay from 10 to 34 days. There was no mortality in the first postoperative month. Median survival after IRE was 10.2 months compared to 9.3 months in a matched cohort (hazard ratio $=.54, \mathrm{p}=.053)$. The quality of life was declining slowly. $81 \%$ of time after IRE the Karnofsky performance status was $\geq 70$ and sharp decline occurred approximately 8 weeks before death.

In conclusion, IRE is a safe palliative treatment option for a percentage of patients with locally advanced pancreatic carcinoma. The patients treated with open IRE lived a decent life until 8 weeks before their death. We believe that IRE of pancreatic carcinoma can be regarded as an option, if imaging or explorative laparotomy show that R0 resection in not possible.
\end{abstract}

Key words: pancreas, carcinoma, cancer, irreversible electroporation, ablation

Pancreatic cancer is characterized by a delayed diagnosis resulting in advanced stage, ineffective treatment, and extremely poor survival that remains around $6 \%$ in 5 years [1-4]. Curative resection is possible in less than one fifth of patients $[5,6]$. Treatment options in the remaining majority are limited to chemotherapy or chemoradiotherapy, palliative surgery or interventional procedures, and supportive care [1]. Debulking or non-radical surgical R2 resection in unresectable patients neither prolongs life nor improves its quality [7].

Irreversible electroporation (IRE) is a mini-invasive nonthermal ablation technology that uses short electric pulses of high voltage to increase the permeability of cells and induce cellular death. It has an improved safety profile compared to thermal ablation techniques and there have been initial reports of its benefits in the treatment of locally advanced pancreatic carcinoma in terms of progression-free survival [8-10].

In this study, we evaluated our first experience with IRE in 21 patients with unresectable pancreatic carcinoma without metastatic disease (TNM stage III).

\section{Patients and methods}

This prospective study was performed in accordance with the Declaration of Helsinki and the study protocol was approved by the local Institutional Review Board. All patients included in this study signed an informed consent.

Twenty-one patients with unresectable locally advanced pancreatic carcinoma AJCC stage III who underwent IRE between June 2012 and December 2014 were included in the study. Inclusion criteria: 1) unresectable pancreatic carcinoma 


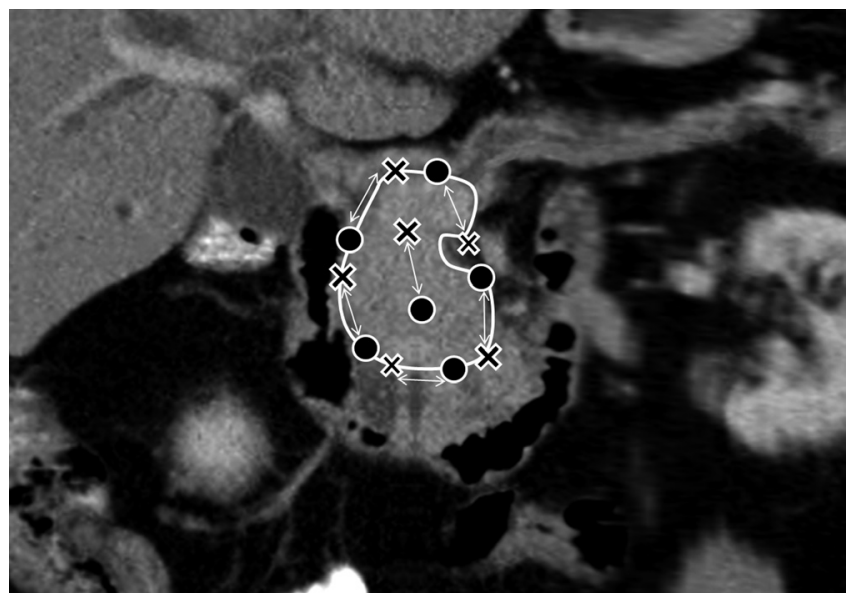

Figure 1. Schematic drawing of placement of two electrodes for IRE. Schematic drawing of placement of two electrodes (activator probe as circle and standard probe as cross) for IRE in a large locally advanced carcinoma of pancreatic head (outlined by white line). The two probes were repositioned several times around the tumor to contain it from all its sides and inside the tumor as well to cover the whole mass.

stage III (without metastatic disease), 2) tumor size $\leq 6.5 \mathrm{~cm}$ in axial plane, 3 ) good performance status (Karnofsky performance status $\geq 80$ ) $[10]$.

Table 1. Characteristics of patients undergoing irreversible electroporation (IRE) and the matched cohort.

\begin{tabular}{lccc}
\hline Characteristics & $\begin{array}{c}\text { IRE } \\
(\mathrm{n}=21)\end{array}$ & $\begin{array}{c}\text { matched cohort } \\
(\mathrm{n}=32)\end{array}$ & $p$ value \\
\hline age (years) & $68.2 \pm 8.4$ & $65.2 \pm 8.7$ & 0.22 \\
male gender & $10(48 \%)$ & $22(69 \%)$ & 0.10 \\
Comorbidity & & & \\
Charlson Comorbidity Index & $3(2-5)$ & $3(2-6)$ & 0.18 \\
hypertension & $12(57 \%)$ & $16(50 \%)$ & 0.61 \\
diabetes & $8(38 \%)$ & $12(38 \%)$ & 0.97 \\
chronic pancreatitis & $6(29 \%)$ & $6(16 \%)$ & 0.40 \\
hyperlipoproteinemia & $6(29 \%)$ & $5(19 \%)$ & 0.26 \\
coronary artery disease & $2(10 \%)$ & $7(22 \%)$ & 0.24 \\
tumor duplicity & $1(5 \%)$ & $3(10 \%)$ & 0.53 \\
Location of the tumor & & & 0.81 \\
head & $17(81 \%)$ & $24(75 \%)$ & \\
body & $3(14 \%)$ & $5(16 \%)$ & \\
tail & $1(5 \%)$ & $3(10 \%)$ & \\
Tumor size & & & \\
maximum diameter in axial & $38.2 \pm 11.5$ & $37.3 \pm 13.9$ & 0.80 \\
plane (mm) & & & 0.43 \\
Histology & $16(76 \%)$ & $22(69 \%)$ & \\
ductal adenocarcinoma & $2(10 \%)$ & $2(6 \%)$ & \\
mucinous adenocarcinoma & $1(5 \%)$ & $1(3 \%)$ & \\
acinary adenocarcinoma & 0 & $5(16 \%)$ & \\
dedifferentiated & $2(10 \%)$ & $2(6 \%)$ & \\
not specified & & & \\
\hline
\end{tabular}

The patients' characteristics are listed in Table 1. Five patients received neoadjuvant chemotherapy (gemcitabine monotherapy, capecitabine plus oxaliplatin combination) [1].

Surgical and IRE technique. IRE was performed 9 weeks (range 2 to 63) after the diagnosis was established. Percutaneous approach was used in two patients and open (intraoperative) approach in 19 patients. Open IRE was combined with the following procedures in four patients: gastroenteroanastomosis (GEA), GEA and cholecystectomy, hepaticojejunoanastomosis (HJA), and cholecystectomy.

After subcostal laparotomy, standard surgical approaches were used to visualize the pancreas with the tumor and to assess its extent and exclude peritoneal seeding. For IRE, the Nanoknife system (Angiodynamics Inc., Quennsbury, NY, USA) with two electrodes (activator and standard probe) was used. The electrodes were placed in the pancreas on the rim of the tumor about 1.5 to $2 \mathrm{~cm}$ apart according to the treatment planning software and the correctness of their placement was verified by a conductivity test. Special care was devoted to avoid an injury to the vascular structures and the pancreatic duct. The pulse settings were: voltage $=2200$ - $3000 \mathrm{~V}$, current $=30-40 \mathrm{~A}$, pulse duration $=90 \mathrm{~ms}, 70$ pulses per second [10]. The probes were repositioned several times around the tumor to contain it from all sides (Figure 1). In larger masses $(>3.5 \mathrm{~cm})$, the electrodes were also placed in the tumor to affect the whole mass and extended in two depths in the tumor. Placement of the electrodes was always performed by the same person with extensive experience in ablation techniques. The duration of one ablation cycle was about $1-4$ minutes. The two electrodes cost 4800Eur altogether.

The duration of the open IRE was measured from the time of the first incision to the last skin suture. In the percutaneous IRE, it was the time from the first puncture of the abdominal wall to the withdrawal of the last electrode. Laboratory and hematology tests were performed before and after the procedure.

Follow-up. The patients were followed-up at regular intervals. Seven patients received chemotherapy after IRE. Postoperative CT of the abdomen was performed $1-2$ months after the procedure, unless required earlier for clinical reasons. If a patient had not appeared for more than three months, his general practitioner was contacted. The quality of life (Karnofsky score) on a scale from 0 (death) to 100 (normal life) was assessed at each clinical visit.

Control group. The patients undergoing IRE were compared with matched controls $(n=32$, propensity score matching based on age and size of the tumor on a 1.5:1 basis) with locally advanced pancreatic carcinoma AJCC stage III, that had undergone surgery (explorative laparotomy, non-radical resection, bypass surgery, cholecystectomy, biopsy) or percutaneous biopsy only ( 3 patients matched to 2 patients with percutaneous IRE) with or without chemotherapy [9].

Statistical analysis. Statistical tests were performed using Prism 5.0 (GraphPad Software, San Diego, USA). To test for 
statistical significance, we used t-test, Mann-Whitney test, and Fisher test. Kaplan-Meier estimator and log-rank (MantelCox) test were used for survival analysis. A p-value below .05 was considered significant. The graphs were plotted in Prism 5.0 and Microsoft Excel 2010.

\section{Results}

The average duration of the open IRE was $79 \pm 23 \mathrm{~min}$ and in patients where it was combined with other surgical procedures, it was prolonged by 4 minutes $(83 \pm 32 \mathrm{~min})$. The percutaneous IRE in the two patients took 24 and $28 \mathrm{~min}$. If no complications occurred, the patients were discharged after 10 days (range 4 to 22 days) compared to 34 days (range 10 to 58 days, $p=.026$ ) in five patients with complications listed in Table 2. Altogether, a patient from the IRE group spent 23 days (range 6 to 150 days) in hospital compared to 26 days (2 to 166 days, $p=.35$ ) in the matched cohort, which amounted to $8 \%$ (range 1 to $94 \%$ ) compared to $16 \%$ (range 1 to $100 \%, p=.092$ ) of follow-up time. Median survival after IRE was 10.2 months compared to 9.3 months in the control group (hazard ratio $=$ $.54, p=.053$, Figure 2 ). There was zero mortality in IRE patients in the first postoperative month, but the presence of complications resulted in reduced survival (7.1 vs. 13.6 months, hazard ratio $=2.3, p=.24)$. The quality of life after IRE was declining slowly, $81 \%$ (interquartile range $65 \%$ to $98 \%$ ) of time after IRE compared to $74 \%(14 \%$ to $88 \%, p=.076)$ in the control group with the performance status $\geq 70$. Sharp decline occurred approximately eight weeks before death (Figure 3 ).

In 19 patients, who underwent CT of the abdomen one to two months after IRE, we observed a combination of the following changes: peripancreatic edema $(n=9)$, pancreatic or peripancreatic necrosis $(n=6)$, peripancreatic or supramesocolic inflammatory infiltrate $(n=4)$, enlarged lymph nodes $(n=4)$, carcinosis with ascites $(n=4)$, extension

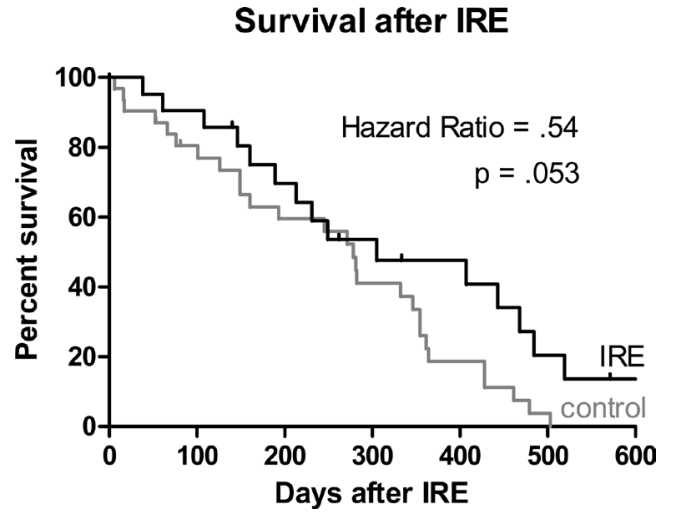

Figure 2. Kaplan-Meier survival plot of patients after IRE.Kaplan-Meier plot shows survival of patients after IRE (10.2 months, black line) compared to a matched cohort ( 9.3 months, hazard ratio $=.54, p=.053$, grey line).

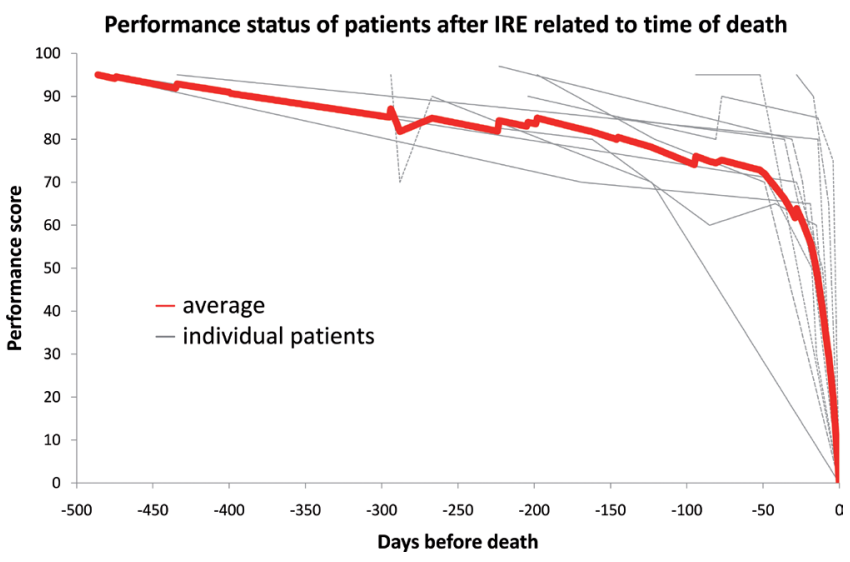

Figure 3. Quality of life in patients after IRE. Quality of life in patients after IRE expressed as Karnofsky performance score related to the time of death shows that sharp decline occurred around 8 weeks before the death.

Table 2. Complications of IRE in five patients.

\begin{tabular}{lllc}
\hline IRE type & Complication & Treatment & Hospital stay \\
\hline percutaneous & biliary peritonitis, cholangoitis, liver abscesses & revision, antibiotics & 48 days \\
percutaneous & pancreatic fistula & stoma bag, antibiotics & 14 days \\
open & bleeding & revision & 10 days \\
open & peripancreatic abscess & percutaneous drainage, antibiotics & 34 days \\
open & fistula and abscess in the abdominal wall & incision, drainage, antibiotics & 58 days \\
\hline
\end{tabular}

IRE: irreversible electroporation

Table 3. Laboratory and hematology parameters in patients before and after irreversible electroporation (IRE).

\begin{tabular}{lcccccc}
\hline Parameter & Unit & Reference value & Before IRE & After IRE & Time after IRE & p value \\
\hline CA 19-9 & $\mathrm{kIU} / \mathrm{L}$ & $0-37$ & $132(0-19000)$ & $172(0-96000)$ & $1-2$ months & .873 \\
Serum amylase & $\mu \mathrm{kat} / \mathrm{L}$ & $0-0.88$ & $0.47(0-1.65)$ & $0.21(0-2.78)$ & $1-2$ weeks & .087 \\
C-reactive protein & $\mathrm{mg} / \mathrm{L}$ & $0-10$ & $13.6(0-114)$ & $54.7(14.3-224.2)$ & $1-2$ weeks & .016 \\
Neutrophile count & $10^{9} / \mathrm{L}$ & $1.8-7$ & $5.2(0.8-11.5)$ & $6.0(3.0-15.9)$ & $1-2$ weeks & .204 \\
Leukocyte count & $10^{9} / \mathrm{L}$ & $4-10$ & $7.8(3.6-15.1)$ & $8(4.6-18.2)$ & $1-2$ weeks & .226 \\
\hline
\end{tabular}


of the tumor into the liver $(n=1)$, and no changes in four patients. Disease progression occurred in eight patients and in the rest the stage remained unchanged. In general, the size of the tumor did not change $(39 \pm 10 \mathrm{~mm}$ vs. $39 \pm 14 \mathrm{~mm}$, $p=.65$ ). In five patients, it decreased in size by $\geq 10 \mathrm{~mm}$. The changes in laboratory and hematology parameters are summarized in Table 3.

\section{Discussion}

This is the first study that attempted to evaluate intraoperative in-situ IRE in patients with even larger pancreatic tumors (up to $6.1 \mathrm{~cm}$ ), which are in some studies beyond the indication criteria that recommend to treat tumors no greater than $3.5 \mathrm{~cm}$ [10]. In comparison with a study by Martin et al., who reported an overall survival improvement from 13 to 20 months compared to a matched cohort of patients, the median survival of our patients was 10.2 months [9]. This can be explained by the fact that half of our patients were not preselected by progression-free interval during neoadjuvant chemotherapy. Only $33 \%$ of them received post IRE chemotherapy and none of them received a surgical resection of the tumor with margin accentuation by IRE. The median survival in our patients was comparable with the matched cohort and also with the standard 6-11 months reported in phase III trials with chemotherapy alone or in combination with radiotherapy $[2,3]$. The fact that survival and the presence of complications were independent of the time of IRE confirms that the experience of the specialist was adequate from the beginning.

Unlike thermal ablation techniques, IRE poses a minimal risk of damaging adjacent vascular structures or inducing pancreatitis [11]. This is underlined by the fact that there was no significant change in serum amylase levels in our patients in the first and second postoperative week compared to the preoperative levels. This observation is consistent with findings by Bower et al. who showed that the serum levels of pancreatic enzymes show only a transient mild increase early in the first postoperative week $[11,12]$. Even though there was no laboratory evidence of acute pancreatitis after IRE, postoperative CT showed signs of various degrees of peripancreatic inflammation in the majority of patients. Increased levels of C-reactive protein postoperatively were in line with other abdominal surgical procedures. Patients after IRE lived a decent life which deteriorated substantially about eight weeks before their death due to progressive disease.

In IRE patients, CA 19-9 level decreased in six patients only, as it usually does after successful R0 resection of the tumor, but in general its level remained unchanged [13]. Non-production of CA 19-9 that is associated with worse prognosis was found in three (15\%) patients [14].

Although percutaneous IRE of pancreatic carcinoma is reportedly a safe procedure, our experience with two patients both of whom had complications was discouraging and was therefore abandoned $[15,16]$. In practice, it is sometimes difficult to find a safe path to perform even a biopsy of a pancreatic tumor, let alone precise placement of several electrodes. Furthermore, there are obvious advantages of the open IRE: full visual control of the electrode placement that avoids injury to adjacent structures including the stomach and bowel loops and allows some degree of angulation, better control of bleeding, and the opportunity to perform further surgical procedures such as GEA, HJA, cholecystectomy, celiac plexus block, or biopsy at the same time [17]. Although pancreatic carcinoma can be treated by IRE irrespective of its location, there are no reports of its use in tumors arising from ectopic pancreatic tissue [18].

Apart from IRE, other local ablation techniques have been previously used in the treatment of unresectable pancreatic cancer. Cryoablation has reportedly a low complication rate and offers minimal improvement in the survival with a median of 8.4 months, but also has effective pain control with better patient performance [19]. Further options include radiofrequency, microwave ablation, photodynamic therapy and high frequency focused ultrasound. These mini-invasive ablation techniques can be further combined with other types of palliative therapy such as chemotherapy, tele- or brachytherapy to maximize the effort to improve survival and the quality of life [9]. The complication rate could be further reduced by pharmacological suppression of the pancreatic secretion using somatostatin analogue and by filling the holes created by the probes by thrombin foam [19].

In conclusion, intraoperative IRE is a relatively short and safe treatment option for a percentage of patients with locally advanced pancreatic carcinoma and it can be combined with other palliative surgical procedures. There is no laboratory evidence of acute pancreatitis after IRE even though postoperative CT shows signs of various degrees of peripancreatic inflammation in the majority of patients and postoperative markers of inflammation are consistent with other abdominal surgical procedures. Although we could not prove any advantage in the overall survival in comparison with matched controls, patients after IRE lived a decent life until about eight weeks before their death. We believe that IRE of pancreatic carcinoma can be regarded as an option if imaging or explorative laparotomy show that the carcinoma cannot be safely resected.

Study limitations. This study was performed with a limited number of patients. The study group was inhomogeneous in terms of the treatment (chemotherapy, days between the diagnosis and IRE), location and size of the tumor. Another reason why our study must be interpreted with some caution, is the absence of randomization which was replaced by a matched cohort. The original design as a randomized study was soon abandoned, because patients in the control group would be deprived of the opportunity to undergo a novel treatment option that might improve the rest of their life.

Acknowledgements: This study was supported by IGA NT/13263-4 and the Ministry of Health No. RVO VFN 64165. 


\section{References}

[1] TEMPERO MA, ARNOLETTI JP, BEHRMAN S, BEN-JOSEF E, BENSON AB, et al. Pancreatic Adenocarcinoma. J Natl Compr Canc Netw 2010; 8: 972-1017.

[2] CARDENES HR, CHIOREAN EG, DEWITT J, SCHMIDT M, LOEHRER P. Locally Advanced Pancreatic Cancer: Current Therapeutic Approach. The Oncologist 2006; 11: 612-623. http://dx.doi.org/10.1634/theoncologist.11-6-612

[3] PULEO F, MARECHAL R, DEMETTER P, BALI M-A, CALOMME A, et al. New challenges in perioperative management of pancreatic cancer. World J Gastroenterol 2015; 21 : 2281-2293. http://dx.doi.org/10.3748/wjg.v21.i8.2281

[4] SIEGEL R, MA J, ZOU Z, JEMAL A. Cancer statistics, 2014. CA Cancer J Clin 2014; 64: 9-29. http://dx.doi.org/10.3322/ caac. 21208

[5] GEER RJ, BRENNAN MF. Prognostic indicators for survival after resection of pancreatic adenocarcinoma. Am J Surg 1993; 165: 68-73. http://dx.doi.org/10.1016/S0002-9610(05)80406-4

[6] LI D, XIE K, WOLFF R, ABBRUZZESE JL. Pancreatic cancer. The Lancet 2004; 363: 1049-1057. http://dx.doi.org/10.1016/ S0140-6736(04)15841-8

[7] Koninger J, WENTE MN, MULLER-STICH BP, DI MOLA FF, GUTT CN, et al. R2 resection in pancreatic cancer--does it make sense? Langenbecks Arch Surg 2008; 393: 929-934. http://dx.doi.org/10.1007/s00423-008-0308-4

[8] MARTIN RCG, MCFARLAND K, ELLIS S, VELANOVICH V. Irreversible Electroporation Therapy in the Management of Locally Advanced Pancreatic Adenocarcinoma. J Am Coll Surg 2012; 215: 361-369. http://dx.doi.org/10.1016/j. jamcollsurg.2012.05.021

[9] MARTIN RCG, MCFARLAND K, ELLIS S, VELANOVICH V. Irreversible Electroporation in Locally Advanced Pancreatic Cancer: Potential Improved Overall Survival. Ann Surg Oncol 2012; 20: 443-449. http://dx.doi.org/10.1245/s10434-012-2736-1

[10] MARTIN RCG. Irreversible Electroporation of Locally Advanced Pancreatic Head Adenocarcinoma. J Gastrointest Surg 2013; 17: 1850-1856. http://dx.doi.org/10.1007/s11605-0132309-Z
[11] BOWER M, SHERWOOD L, LI Y, MARTIN R. Irreversible electroporation of the pancreas: Definitive local therapy without systemic effects. J Surg Oncol 2011; 104: 22-28. http:// dx.doi.org/10.1002/jso.21899

[12] JOSE A, SOBREVALS L, IVORRA A, FILLAT C. Irreversible electroporation shows efficacy against pancreatic carcinoma without systemic toxicity in mouse models. Cancer Lett 2012; 317: 16-23. http://dx.doi.org/10.1016/j. canlet.2011.11.004

[13] PARK JK, PAIK WH, RYU JK, KIM Y-T, KIM YJ, et al. Clinical Significance and Revisiting the Meaning of CA 19-9 Blood Level Before and After the Treatment of Pancreatic Ductal Adenocarcinoma: Analysis of 1,446 Patients from the Pancreatic Cancer Cohort in a Single Institution. PLoS ONE 2013; 8: e78977. http://dx.doi.org/10.1371/journal.pone.0078977

[14] HAYMAN AV, STOCKER SJ, BAKER MS, BENTREM DJ, PRINZ RA, et al. CA 19-9 nonproduction is associated with poor survival after resection of pancreatic adenocarcinoma. Am J Clin Oncol 2014; 37: 550-554. http://dx.doi.org/10.1097/ COC.0b013e318280d5f0

[15] NARAYANAN G, HOSEIN PJ, LIMA CMSR, SUTHAR R, MERCHAN JR, et al. Percutaneous irreversible electroporation (IRE) in the management of pancreatic cancer. J Clin Oncol 2014; 32. http://dx.doi.org/10.1016/j.jvir.2013.12.083

[16] NARAYANAN G, HOSEIN PJ, ARORA G, BARBERY KJ, FROUD T, et al. Percutaneous irreversible electroporation for downstaging and control of unresectable pancreatic adenocarcinoma. J Vasc Interv Radiol 2012; 23: 1613-1621. http:// dx.doi.org/10.1016/j.jvir.2012.09.012

[17] KAWAMATA M, ISHITANI K, ISHIKAWA K, SASAKI H, OTA K, et al. Comparison between celiac plexus block and morphine treatment on quality of life in patients with pancreatic cancer pain. Pain 1996; 64: 597-602. http://dx.doi. org/10.1016/0304-3959(95)00189-1

[18] GRUS T, TRCA S, LAMBERT L, GRUSOVA G, KOVACOVA Z. Extensive pancreatic heterotopia in distal esophagus mimicking esophageal malignancy. Eur Surg 2015; 47: 206-209. http://dx.doi.org/10.1007/s10353-015-0326-8

[19] XU K, NIU L, YANG D. Cryosurgery for pancreatic cancer. Gland Surg 2013; 2: 30-39. 